\title{
Chagas Disease in Europe: a long way to go
}

Spinello Antinori, Mario Corbellino

${ }^{1}$ Department of Biomedical and Clinical Sciences “Luigi Sacco”, University of Milano, Italy; ${ }^{2}$ III

Division of Infectious Diseases, ASST Fatebenefratelli Sacco, Luigi Sacco Hospital, Milano, Italy

Correspondence to:

Prof. Spinello Antinori

Department of Biomedical and Clinical Sciences Luigi Sacco

Università degli Studi di Milano

Via GB Grassi, 74

20157 Milano, Italy

Tel. $N^{\circ}+390250319765$

Fax $N^{\circ}+390250319758$

e-mail: spinello.antinori@unimi.it

Word counts: 344

Figure : 1 
We appreciate the comments by Ramos to our review on Chagas' disease (CD) in Europe [1,2]. We congratulate the Author and his group for the efforts made to identify people of Latin American (LA) origin who are infected by Trypanosoma cruzi and unaware of it using creative strategies.. Indeed, our intention was precisely to raise the attention of the medical community in Europe working out our specialty (i.e., Infectious Diseases) to the growing problem of CD in all its protean manifestations (Figure 1). Our wake up call was especially, but not exclusively, addressed to the Countries having the highest number of LA immigrants.

We fully agree with Ramos’ last sentence when he says that CD is an “opportunity” for Physicians with different specializations, but we believe the disease should be more appropriately considered a “call to army”.

However, the Author’s idea to offer a serologic screening for CD to LA caregivers of elderly patients who are hospitalized in Medicine wards should be applauded and possibly extended to Italy and other countries where this phenomenon is increasingly encountered.

Nevertheless, it is clear that individual actions that are in place also in Italy, such as those conducted in the last years in Milan and Bergamo [3,4] thanks to "Doctors without Borders", are not sufficient per se: it is imperative that "institutional” initiatives driven by the National Health Care Systems are rapidly implemented.

In this regard the Spanish model of CD screening should be adopted by the rest of the European countries (5). Although the efficacy of benznidazole and nifurtimox the two drugs currently employed in the treatment of CD is far from optimal for patients with the chronic indeterminate stage of the infection, it has clearly been demonstrated that the earlier the treatment is begun, the better is the response.

In addition, up to $98 \%$ of infants with congenital CD who are treated precociously go on to acquire negative T. cruzi serology and parasitemia. 
Finally it is hopeful that pharmaceutical companies may be conviced to develop novel agents s for the treatment of this neglected disease.

\section{Conflicts of interest}

Prof Spinello Antinori . None

Dr Mario Corbellino : None 


\section{References}

1. Ramos JM. Chagas diseases: opportunity for internists. Eur J Intern Med 2017, http://dx.doi.org/10.1016/j.ejim.2017.09.005

2. Antinori S, Galimberti L, Bianco R, Grande R, Galli M, Corbellino M. Chagas disease in Europe: a review for the internist in the globalized world. Eur J Intern Med 2017; 43:6-15.

3. Antinori S, Grande R, Bianco R, Traversi L, Cogliati C, Torzillo D, et al. High frequency of adverse reactions and discontinuation with benznidazole treatment for chronic Chagas disease in Milan, Italy. Clin Infect Dis 2015;60:1873-5.

4. Repetto EC, Zachariah R, Kumar A, Angheben A, Gobbi F, Anselmi M, et al. Neglect of a neglected disease in Italy: the challenge of access-to-care for Chagas disease in Bergamo area. Plos Neglect Trop Dis 2015;9(9):e0004103.

5. Generalitat de Catalunya-Department de Salut. Protocol for screening and diagnosing Chagas disease in pregnant Latin American women and their newborns. Barcelona 2010; 144.http://canalsalut.gencat.cat/web/.content/home_canal_salut/professionals/temes_de_salut /chagas/documents/arxius/chagas_angles.pdf (last accessed September 15, 2017) 
Figure legend: Clinical algorithm with bidirectional involvement between different specialists and Infectious Diseases specialist for the management of Latin American people with suspected or confirmed Chagas disease

LA = Latina American: PCR= polymerase chain reaction

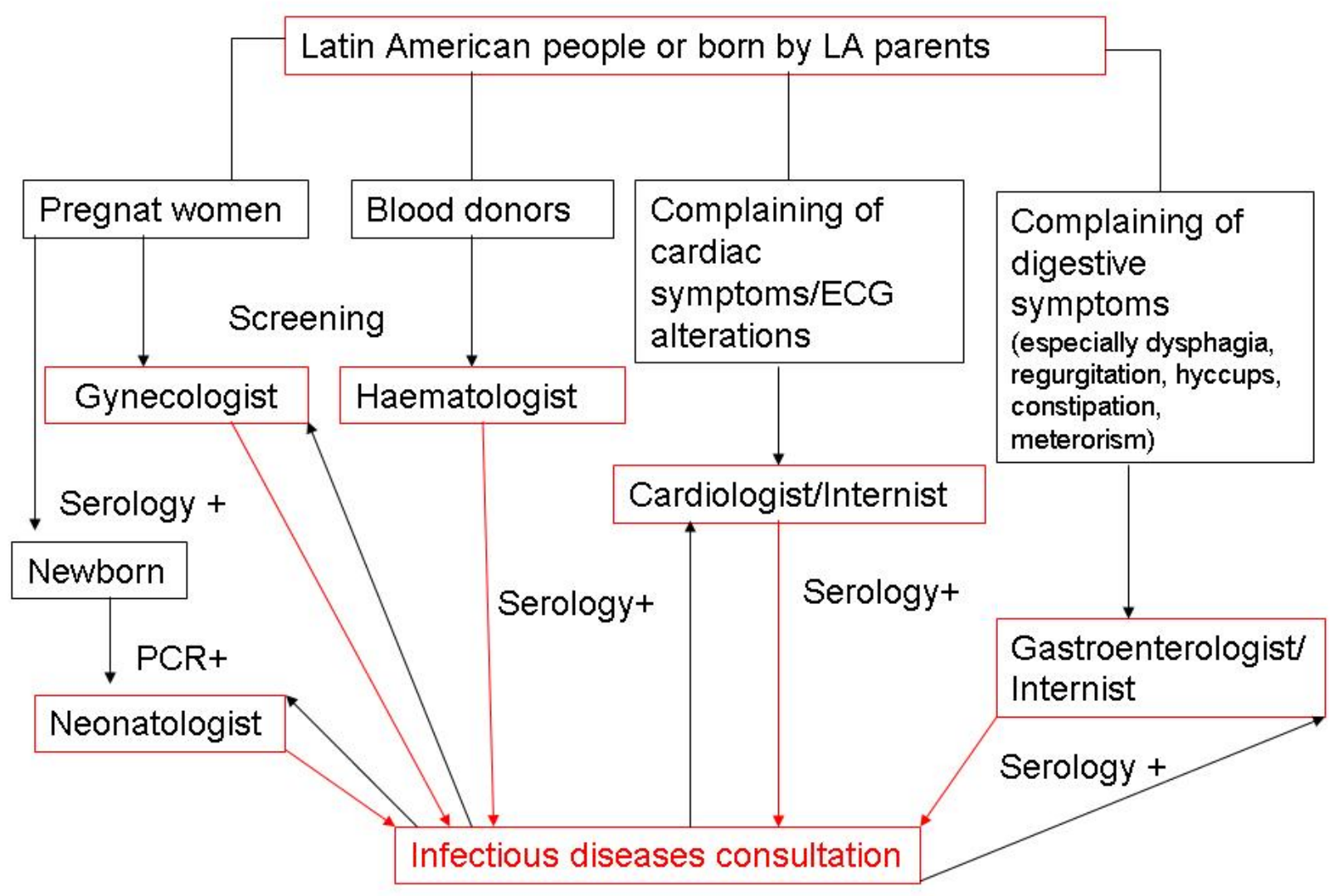

